\title{
High-repetition-rate white-light pump-probe spectroscopy with a tapered fiber
}

\author{
M. Punke, F. Hoos, ${ }^{*}$ C. Karnutsch, and U. Lemmer \\ Light Technology Institute and Center for Functional Nanostructures (CFN), Universität Karlsruhe (TH), \\ Kaiserstrasse 12, D-76131 Karlsruhe, Germany \\ N. Linder and K. Streubel \\ OSRAM Opto Semiconductors GmbH, Wernerwerkstrasse 2, D-93049 Regensburg, Germany
}

\begin{abstract}
Received December 13, 2005; accepted January 10, 2006; posted January 25, 2006 (Doc. ID 66614)
We have realized a $76 \mathrm{MHz}$ white-light differential transmission spectroscopy system. The technique employs a Ti:sapphire laser oscillator and a tapered fiber to generate a white-light continuum spanning almost the full visible to near-infrared spectral range. Using acousto-optical modulation and subsequent lock-in detection, transient relative transmission changes as small as $10^{-5}$ are detected. The method is applied to study the ultrafast gain dynamics of the active layer of a vertical-external-cavity surface-emitting laser based on a multiple-quantum-well structure. (C) 2006 Optical Society of America

OCIS codes: $320.7130,320.7140,320.7150$.
\end{abstract}

The investigation of charge-carrier dynamics in semiconductor heterostructures is one of the most important applications of femtosecond pump-probe spectroscopy. ${ }^{1,2}$ Compared with pump-probe spectroscopy using a single wavelength, the use of a white-light probe pulse allows for much more insight into the underlying processes. For the investigation of semiconductor thin films for laser applications this is of particular importance, since the gain spectrum determines the device operation. The conventional approach for multiwavelength differential transmission spectroscopy is the use of an amplified femtosecond laser system to create a high-energy pump pulse that is focused onto a nonlinear medium to generate a white-light continuum. The drawbacks of this approach are the complexity and the high costs of such femtosecond laser amplifier systems. In another approach the use of an extremely short pulse ( $5 \mathrm{fs}$ ) as an ultrabroadband probe beam source was demonstrated. ${ }^{3}$

Recently, white-light continuum generation using unamplified Ti:sapphire laser oscillators was demonstrated with microstructured as well as tapered fibers. ${ }^{4,5}$ Tapered fibers are single-mode optical fibers drawn over a flame to form a region where the fiber diameter is significantly decreased over a certain length. Light of an incoming laser pulse is concentrated in the taper region. The main nonlinear effects for the white-light generation process are self-phase modulation, soliton splitting, Raman scattering, and four-wave mixing. ${ }^{6}$

This remarkable simplification of the white-light generation process also opens up possibilities for less complex but powerful setups for pump-probe spectroscopy. Here we demonstrate for the first time to our knowledge the use of $76 \mathrm{MHz}$ spectroscopy by means of the white light generated in a tapered fiber to study the gain dynamics in a semiconductor heterostructure.

The structure under investigation is the gain region of a vertical-external-cavity surface-emitting la- ser (VECSEL). These devices have recently emerged as an important class of semiconductor lasers because of their ability to generate near-transformlimited subpicosecond pulses at high average power and repetition rates of many gigahertz. ${ }^{7,8}$ Their differential gain, gain bandwidth, and peak gain wavelength are a matter of current research. ${ }^{9-11}$ Using our novel spectroscopic setup, we report ultrafast measurements of the carrier dynamics in a red-emitting VECSEL multiple-quantum-well (MQW) gain element. The structure is shown in the inset of Fig. 4 below. It consists of an AlGaInP MQW structure comprising 32 strained GaInP QWs. These are arranged in eight groups containing four $\mathrm{QWs}$ and five $\left(\mathrm{Al}_{0.5} \mathrm{Ga}_{0.5}\right)_{0.52} \mathrm{In}_{0.48} \mathrm{P}$ barrier layers each. The concluding AlInP capping layer acts as a barrier for the photogenerated charge carriers to prevent nonradiative surface recombination. ${ }^{12}$

The experimental pump-probe setup is shown schematically in Fig. 1. A Kerr-lens mode-locked Ti:sapphire laser (center wavelength tuning range $770-850 \mathrm{~nm}$, pulse width $60 \mathrm{fs}$, repetition rate $76 \mathrm{MHz}$; Del Mar Ventures, San Diego) is used as the

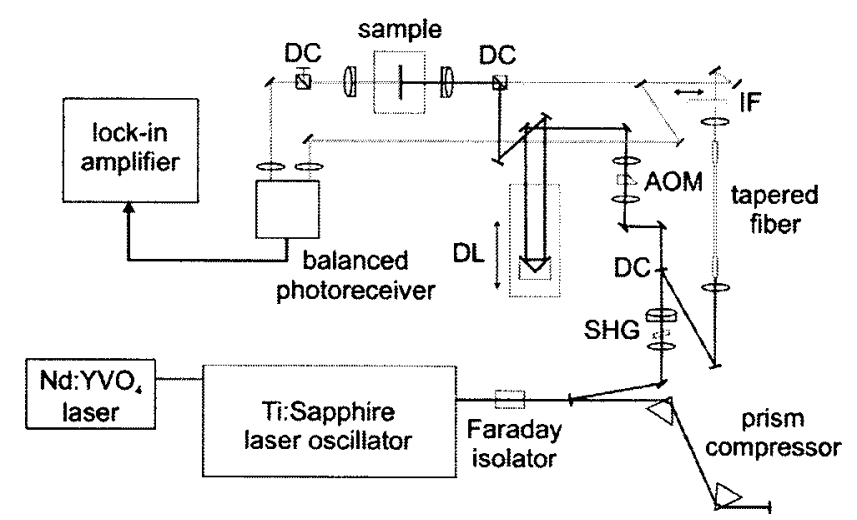

Fig. 1. Schematic diagram of the experimental setup. AOM, acousto-optic modulator; DC, dichroic mirror; DL, delay line; IF, variable interference filter; SHG, secondharmonic generation. 
fundamental laser source. The output of the femtosecond laser passes through a Faraday isolator to avoid backreflections from the subsequent nonlinear crystal and the tapered fiber. The chirp introduced by the optical elements in the beam path is precompensated by using a double-pass prism compressor. One part of the laser pulse is converted into a $400 \mathrm{~nm}$ pump pulse by second-harmonic generation in a nonlinear crystal (LBO). The residual beam at $800 \mathrm{~nm}$ is separated by a dichroic mirror and focused into the tapered fiber arrangement (NT\&C, Marnheim, Germany) by using a micropositioning stage and a $10 \times$ microscope objective. A single probing wavelength is selected from the resulting white-light continuum at the fiber output by the use of a variable interference filter (FWHM $\sim 13 \mathrm{~nm}$ ).

The pump beam is modulated at $115 \mathrm{kHz}$ by an acousto-optic modulator (AOM) to allow for a highly sensitive lock-in detection scheme. The beam then passes through an optical delay line consisting of a $150 \mathrm{~mm}$ linear positioning stage and a retroreflector. Thus a delay time between pump and probe pulses of more then $600 \mathrm{ps}$ is achievable. A dichroic mirror is used to combine the pump and the probe beam. In this collinear arrangement, the two beams are focused by the same achromatic lens $(f=100 \mathrm{~mm})$, resulting in nearly circular spots on the sample. The spot diameters are $\sim 70$ and $\sim 50 \mu \mathrm{m}$ for the pump and the probe beams, respectively. A filter cube comprising a dichroic mirror and a blocking filter suppresses the pump light passing through the sample. For an increased signal-to-noise ratio, a reference beam is separated before the sample and sent onto a balanced photoreceiver. ${ }^{13}$ The output signal is fed into a lock-in amplifier.

From the resulting signal $\Delta T$ we can calculate the differential transmission $\Delta T / T_{0}=\left(T-T_{0}\right) / T_{0}$, where $T$ is the transmission influenced by the pump beam and $T_{0}$ is the transmission without the pump beam. The uninfluenced transmission $T_{0}$ is measured by blocking the pump beam and simultaneously detecting the signal from the probe beam.

We employ a single-mode quartz fiber (Corning SMF28) with a core diameter of $9 \mu \mathrm{m}$ for the generation of the probe continuum. The fiber is tapered down to $1.85 \mu \mathrm{m}$ in the $8 \mathrm{~cm}$ long waist region (see inset, Fig. 2). Figure 2 shows output spectra of the fiber as a function of the input power. The output spectrum broadens with increased input power. The resulting spectrum can span the range from 450 to $1000 \mathrm{~nm}$. Longer input wavelengths cause a broader spectrum due to the dispersion parameters of the fiber. A flat spectral characteristic is achieved when shorter wavelengths are used. For achieving the broadest spectra, chirp-compensated input pulses have to be used.

For a noise analysis of the system, the signal of the photoreceiver was recorded with a digital sampling oscilloscope for a fast Fourier transformation analysis. Compared with the case of an $800 \mathrm{~nm}$ probe beam derived directly from the laser oscillator, the noise level in the white-light continuum is significantly enhanced. ${ }^{14}$ We observe an enhancement of

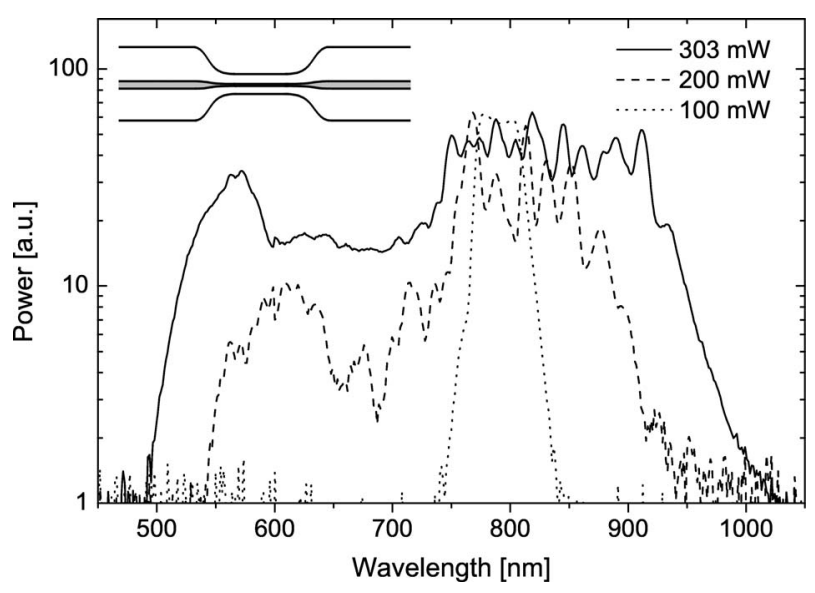

Fig. 2. Normalized tapered fiber output spectra as a function of the input power. Input wavelength, $789 \mathrm{~nm}$. Inset, scheme of the tapered fiber.

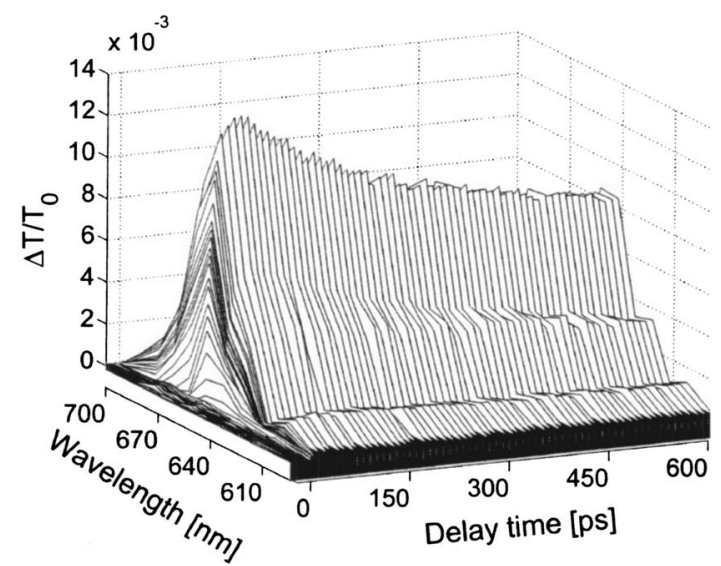

Fig. 3. Differential transmission measurements of the investigated AlGaInP MQW structure. Pump wavelength, $400 \mathrm{~nm}$; pump pulse energy, 150 pJ.

$10-20 \mathrm{dBc} / \mathrm{Hz}$ in the relative intensity noise of spectral portions of the continuum (spectral width $13 \mathrm{~nm}$ ) as compared with the $800 \mathrm{~nm}$ laser light. The spectral dependence between 500 and $800 \mathrm{~nm}$ is not pronounced. The sensitivity of the setup is $10^{-6}$ in $\Delta T / T_{0}$ for pump probe measurements using an $800 \mathrm{~nm}$ probe beam. It is reduced to $10^{-5}$ in $\Delta T / T_{0}$ for the case of measurements using spectrally narrow portions of the white-light continuum.

For the differential transmission measurements of the semiconductor sample, a pump pulse energy of $150 \mathrm{pJ}$ was used. The probe pulse energy was 2 orders of magnitude lower, and the probe wavelengths covered the spectral range from 590 to $700 \mathrm{~nm}$. The delay time between pump and probe pulses was varied over a $600 \mathrm{ps}$ range. The resulting threedimensional data set is shown in Fig. 3. The gain spectrum extends over more than $50 \mathrm{~nm}$ for this pumping condition. The peak gain is observed at a probe wavelength of $650 \mathrm{~nm}$. Here the transmission change amounts to $\Delta T / T_{0}=1.44 \times 10^{-2}$. Because of the good sample quality, large carrier lifetimes of more than 500 ps are measured. Figure 4 compares the maximum transmission changes with a photoluminescence spectrum. The highest gain is found at the high-energy side of the photoluminescence peak. 
In summary, we have presented, for the first time to our knowledge, the use of a white-light continuum output from a tapered fiber for high-sensitivity $76 \mathrm{MHz}$ pump-probe measurements. Since the continuum is directly generated by pulses from a Ti:sapphire laser oscillator, a femtosecond amplifier system is no longer required. Relative differential transmission changes as small as $10^{-5}$ can be detected. The investigation of transmission changes in an AlGaInP MQW structure demonstrates the system's practicability. The reduction of the continuum noise is under investigation. Noise suppression methods such as stabilization of the Ti:sapphire laser and radio-frequency lock-in detection should further improve the sensitivity of the pump-probe system.

This work has been financially supported by the DFG Research Center for Functional Nanostructures (CFN). M. Punke's e-mail address is martin.punke@lti.uni-karlsruhe.de.

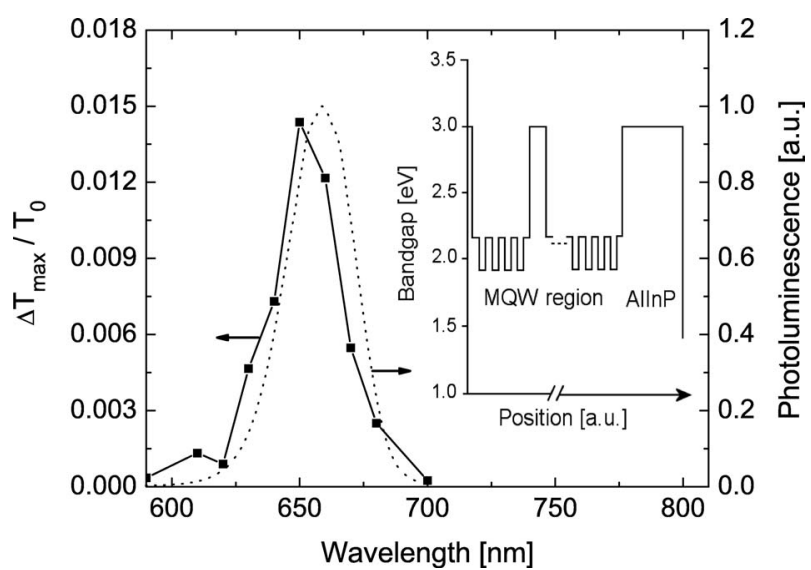

Fig. 4. Maximum of the differential transmission change in comparison with the photoluminescence spectra of the MQW structure. Inset, scheme of the AlGaInP MQW structure.
*Present address, Universität Stuttgart, 4, Physikalisches Institut, Pfaffenwaldring 57, D-70550 Stuttgart, Germany.

\section{References}

1. S. Link, H. A. Duerr, and W. Eberhardt, J. Phys. Condens. Matter 13, 7873 (2001).

2. V. M. Axt and T. Kuhn, Rep. Prog. Phys. 67, 433 (2004).

3. W. Seitz, R. Ell, U. Morgner, and F. X. Kaertner, IEEE J. Sel. Top. Quantum Electron. 9, 4 (2003).

4. J. K. Ranka, R. S. Windeler, and A. J. Stentz, Opt. Lett. 25, 25 (2000).

5. T. A. Birks, W. J. Wadsworth, and P. St. J. Russell, Opt. Lett. 25, 1415 (2000).

6. J. Teipel, K. Franke, D. Tuerke, F. Warken, D. Meiser, M. Leuschner, and H. Giessen, Appl. Phys. B 77, 245 (2003).

7. A. Garnache, S. Hoogland, A. C. Tropper, I. Sagnes, G. Saint-Girons, and J. S. Roberts, Appl. Phys. Lett. 80, 3892 (2002).

8. A. C. Tropper, H. D. Foreman, A. Garnache, K. G. Wilcox, and S. Hoogland, J. Phys. D 37, R75 (2004).

9. M. I. Mueller, C. Karnutsch, J. Luft, W. Schmid, K. Streubel, N. Linder, S. S. Beyertt, U. Brauch, A. Giesen, and G. H. Doehler, in Proceedings of the 29th International Symposium on Compound Semiconductors (Institute of Optics, 2003), p. 427.

10. J. M. Hopkins, S. A. Smith, C. W. Jeon, D. Burns, S. Calvez, M. D. Dawson, T. Jouhti, and M. Pessa, Electron. Lett. 40, 30 (2004).

11. J. E. Hastie, S. Calvez, M. D. Dawson, T. Leinonen, A. Laakso, J. Lyytikaeinen, and M. Pessa, Opt. Express 13, 77 (2005).

12. M. I. Mueller, N. Linder, C. Karnutsch, W. Schmid, K. Streubel, J. Luft, S. S. Beyertt, A. Giesen, and G. H. Doehler, in Proc. SPIE 4649, 265 (2002).

13. J. Vollmann, D. M. Profunser, and J. Dual, Ultrasonics 40, 757 (2002).

14. N. R. Newbury, B. R. Washburn, K. L. Corwin, and R. S. Windeler, Opt. Lett. 28, 944 (2003). 